\title{
Statistical properties of solar Ho flare activity
}

\author{
Linhua Deng ${ }^{1,2,3, *}$, Xiaojuan Zhang ${ }^{1,5}$, Jianmei $\mathrm{An}^{4}$ and Yunfang $\mathrm{Cai}^{1,5}$ \\ ${ }^{1}$ Yunnan Observatories, Chinese Academy of Sciences, Kunming 650216, PR China \\ ${ }^{2}$ Key Laboratory of Geospace Environment, Chinese Academy of Sciences, University of Science \& Technology of China, \\ Hefei 230026, PR China \\ ${ }^{3}$ CAS Key Laboratory of Solar Activity, National Astronomical Observatories, Beijing 100012, PR China \\ ${ }^{4}$ School of Software Engineering, Chongqing University of Arts and Sciences, Chongqing 402160, PR China \\ ${ }^{5}$ University of Chinese Academy of Sciences, Beijing 100049, PR China
}

Received 30 March 2017 / Accepted 23 November 2017

\begin{abstract}
Magnetic field structures on the solar atmosphere are not symmetric distribution in the northern and southern hemispheres, which is an important aspect of quasi-cyclical evolution of magnetic activity indicators that are related to solar dynamo theories. Three standard analysis techniques are applied to analyze the hemispheric coupling (north-south asymmetry and phase asynchrony) of monthly averaged values of solar H $\alpha$ flare activity over the past 49 years (from 1966 January to 2014 December). The prominent results are as follows: (1) from a global point of view, solar $\mathrm{H} \alpha$ flare activity on both hemispheres are strongly correlated with each other, but the northern hemisphere precedes the southern one with a phase shift of 7 months; (2) the long-range persistence indeed exists in solar $\mathrm{H} \alpha$ flare activity, but the dynamical complexities in the two hemispheres are not identical; (3) the prominent periodicities of $\mathrm{H} \alpha$ flare activity are 17 years full-disk activity cycle and 11 years Schwabe solar cycle, but the short- and mid-term periodicities cannot determined by monthly time series; (4) by comparing the non-parametric rescaling behavior on a point-by-point basis, the hemispheric asynchrony of solar $\mathrm{H} \alpha$ flare activity are estimated to be ranging from several months to tens of months with an average value of 8.7 months. The analysis results could promote our knowledge on the long-range persistence, the quasi-periodic variation, and the hemispheric asynchrony of solar $\mathrm{H} \alpha$ flare activity on both hemispheres, and possibly provide valuable information for the hemispheric interrelation of solar magnetic activity.
\end{abstract}

Keywords: solar activity cycle / H $\alpha$ flare index / statistical analysis

\section{Introduction}

Solar flares are the violently expulsive phenomena in the Sun. They are observed in a wide range of wavelengths from decameter radio waves to gamma-rays beyond $1 \mathrm{GeV}$ (Yan et al., 2015). The total energy of a large flare is about $10^{32} \mathrm{erg}$ (Benz, 2008), and some flares can produce an intense effect on space environment (Shen et al., 2011). For example, the first white-light flare observed by Carrington and Hodgson has produced a strong geomagnetic storm. Therefore, solar flares become one of the most attractive scientific issues in solar physics and space weather studies (Shen \& Liu, 2012; Feng et al., 2013). Shibata \& Magara (2011) summarized that solar flares are multi-scale complex phenomena with various temporal and spatial scales in the atmospheric layers from photosphere to corona and beyond.

\footnotetext{
*Corresponding author: 1hdeng@ynao.ac.cn
}

The long-term variations of solar magnetic structures driven by the magnetic field evolution have been monitored systematically, and there is a statistical imbalance in the occurrence of magnetic activity indicators on both hemispheres when averaged over a certain time scale ranging from a year to a solar cycle (Yan et al., 2008; Xie et al., 2012; Ouyang et al., 2017). This phenomenon is known as the north-south asymmetry of solar activity, and the existence of the hemispheric asymmetry in different solar activity indicators observed in the solar atmosphere is well known. A statistical analysis of solar $\mathrm{H} \alpha$ flares from 1975 January to 1999 December has been done by Temmer et al. (2001). They found that there indeed exists a significant hemispheric asymmetry over longer periods, and the dominance of one hemisphere over the other one could persist for more than one cycle. For certain solar cycles there may be evidence that the N-S asymmetry evolves with the solar cycle, whereas in general this is not the case. By studying the north-south asymmetry of soft X-ray flare index in cycles 21-23, Joshi \& Joshi (2004) 
found that there exists a real north-south asymmetry in solar flare activity which is not due to random fluctuations, and the hemispheric asymmetry is strengthened during solar minimum. They also found that the hemispheric asymmetry of soft $\mathrm{X}$-ray flare index has a periodic behavior shifted in phase with respect to the solar cycle, and the significant periods of daily asymmetry time series are around 28.26 days, 550.73 days and 3.72 years. Mendoza \& Velasco-Herrera (2011) studied the mid-term periodicities in sunspot groups and flare index by separating the data into hemispheres and spectral bands, and found that the southern hemisphere dominates in most of the spectral bands for sunspot groups, while the northern hemisphere dominates for all spectral bands for flare index. Moreover, the phase lags (obtained from the cross-correlation analysis) found between the two hemispheres implied that the degrees of coupling in the photosphere for sunspot groups and in the corona for flares are between moderate and strong. Given the global character of the coupling estimation, the three broad levels of coupling are assessed in terms of the phase lags, weak: the lag is larger than the upper limit of the spectral band, strong: the lag is smaller than the lower limit of the spectral band, and moderate: the lag lies between the lower and upper limits of the spectral band. Here, the spectral bands are centered at the power peaks above the red-noise level of the global wavelet spectrum. Zhang et al. (2015) applied a differentially rotating reference system to determine the bestfit annual values of the differential rotation parameters of active longitudes of solar X-ray flares and sunspots in 1977-2012, and found that the rotation of both hemispheres has been speeding up at roughly the same rate since the late 1990s, with the southern hemisphere rotating slightly faster than the northern hemisphere. Joshi et al. (2015) investigated the temporal evolution and north-south asymmetry in the occurrence of soft X-ray flare index during solar cycles 21-23. They found that a slight excess of activity in the northern hemisphere during cycle 21 , while a southern excess clearly prevails for cycles 22 and 23. Their study also revealed a significant hemispheric asymmetry, which exhibits variations with the phases of the solar cycle.

Norton et al. (2014) concluded that hemispheric coupling of solar magnetic indicators is an important issue of their cyclic behaviors that are related to solar dynamo models on the relative importance of meridional circulation. Moreover, statistical investigations of the temporal and spatial distribution of solar magnetic indicators over the whole disk and their evolutional relationship along the solar cycles are of fundamental importance, leading to a better understanding of the physical origin and secular evolution of solar magnetism (Ermolli et al., 2014; Deng et al., 2016). So far, the physical causes of hemispheric similarities and distinctions are not fully understood, but the randomness in the formation process of poloidal fields and the spatio-temporal asymmetry of meridional flow could play an important role in the hemispheric coupling of solar magnetic indicators (Goel \& Choudhuri, 2009; Shetye et al., 2015). Svalgaard \& Kamide (2013) suggested that the association of multiple peaks of solar activity when separated by two hemispheres with different times of polar field reversals is an intrinsic characteristic of solar cycle, and found that the asymmetric polar field reversals are simply a consequence of the north-south asymmetry of solar activity. Kilcik et al. (2010) pointed out that statistical analysis of solar flare activity (such as periodic variation, hemispheric difference, and secular trend) could provide information on the physical properties of the Sun, and could better understand the flaring process of energy storage and dissipative mechanism.

The main motivation for this work is to investigate the long-range persistence, the periodic variation, and the hemispheric asynchrony of solar $\mathrm{H} \alpha$ flare activity on both hemispheres for the time interval from 1966 January to 2014 December. Here, we only focus on the long-term temporal and spatial variations of solar $\mathrm{H} \alpha$ flare activity, but its statistical properties in individual solar cycle are not concerned. The paper is organized as follows. The brief description of observational data and analysis methods are described in the next Section. The analysis results obtained from several statistical techniques are presented and compared in Section 3. Finally Section 4 gives the conclusions and discussion.

\section{Observational data and methods of analysis}

\subsection{Observational data}

We focus our analysis on solar $\mathrm{H} \alpha$ flare activity from synoptically chromospheric observations, because of the remarkable archival data. Solar $\mathrm{H} \alpha$ flare activity has been described as flare index, which was first introduced by Josip Kleczek in the middle of last century (Kleczek, 1952). This quantitative indicator is defined as the product of the intensity scale of solar flares observed in the $\mathrm{H} \alpha$ filter and their duration in minutes (Ozguc et al., 2003; Atac \& Ozguc, 1998, 2006). It permits a measure of the short-lived activity of the Sun and allows us to study its short- and long-term evolutions. This twodimensional index is physically suitable enough to describe solar $\mathrm{H} \alpha$ flare activity and could be download from the website of Bogazici University (http://www.koeri.boun.edu.tr/astronomy). From this website one can obtain the daily, monthly, and yearly flare index in the northern and southern hemispheres, and in the whole solar disk. The time interval for both monthly and yearly time series covers from 1966 January to 2014 December, but the daily time series is only from 1976 January 1 to 2014 December 31. For yearly flare index, it is not easy to obtain the hemispheric phase lags and the quasi-periodic variations, since the sampled rate is too low. Zolotova \& Ponyavin (2007) once investigated the north-south synchronization on short time scale using the daily sunspot area data, and found that it is difficult to trace sunspot synchronization on daily time scale. Therefore, we only use the monthly flare index in the two hemispheres to study its statistical properties. Figure 1 displays the monthly distribution of flare index on both hemispheres from 1966 January to 2014 December.

\subsection{Methods of analysis}

In this work, three standard time series analysis approaches, such as the rescaled range $(\mathrm{R} / \mathrm{S})$ analysis, the Lomb-Scargle (LS) periodogram, and the cross-recurrence plot (CRP), are combined to investigate the temporal and spatial distribution of solar $\mathrm{H} \alpha$ flare activity on both hemispheres. To better understand and interpret the analysis 

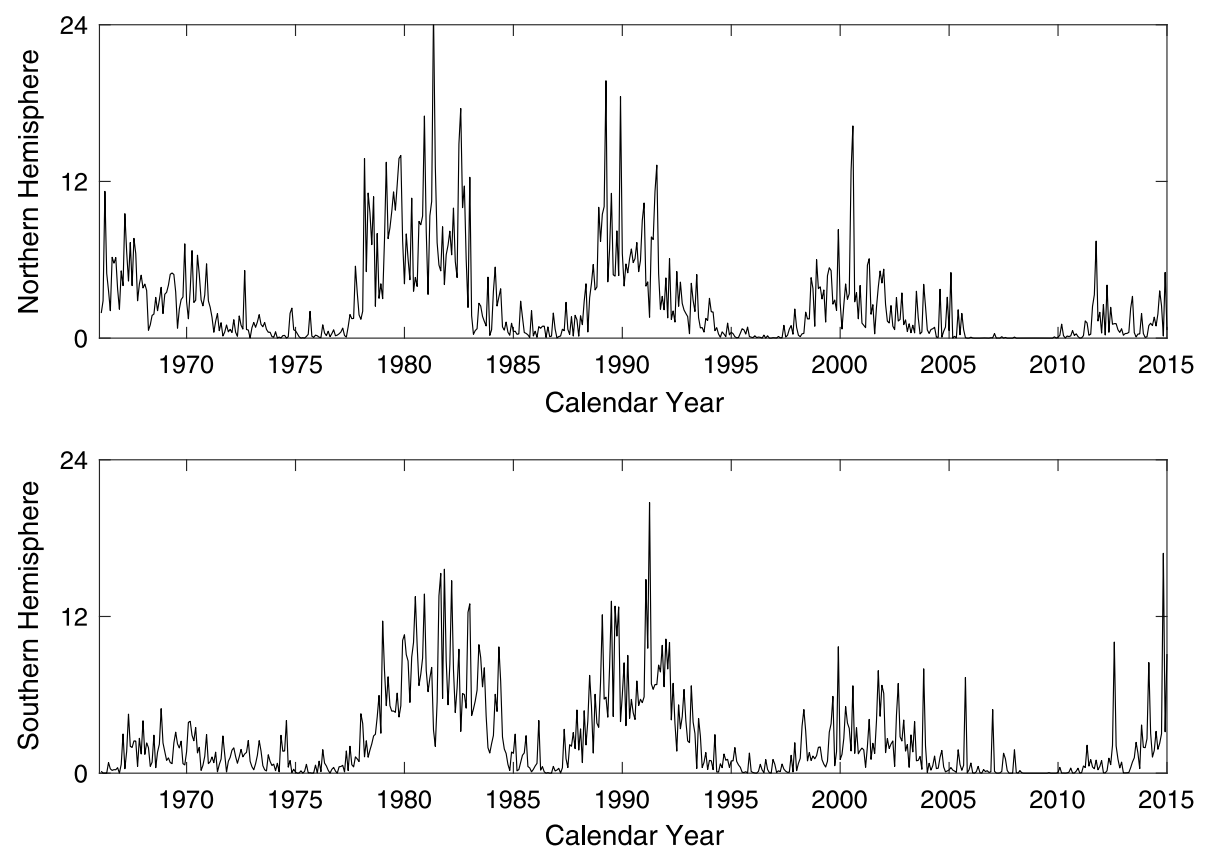

Fig. 1. Monthly distribution of flare index in the northern (upper panel) and southern (lower panel) hemispheres for the time interval from 1966 January to 2014 December.

results presented in Section 3, the brief descriptions of the above three techniques are given below. For details of these three techniques, please refer to the appendices A, B, and C.

The R/S analysis, pioneered by Mandelbrot \& Wallis (1969) following Hurst (1951), is of fundamental importance in describing the statistical properties of a dynamical process and provides an approach for determining whether or not a stochastic time series exhibits long-range persistence (Xapsos \& Burke, 2009). Long-range persistence, also known as longterm memory or long-range correlation, is a common property of records of the variation of spatially or temporarily aggregated variables. In contrast to short-range persistent process, the long-range persistent process exhibits a power-law scaling of the autocorrelation function (Lepreti et al., 2000; Kilic \& Golbasi, 2011).

The LS periodogram is an important time-frequency analysis approach and could be applied to notably improve the results obtainable by the statistical analysis of a time series (Lomb, 1976; Scargle, 1982). Compared with other methods, such as the conventional fast fourier transformation, the LS periodogram has the following advantages: (1) it is appropriate for analyzing the unevenly spaced data, that is, it can mitigate the missing data problem; (2) inclusion of $\tau$ (see Appendix B) makes the periodogram invariant to a shift of the origin of time; and (3) periodic analysis performed by this method is exactly equivalent to the least-square fitting of sine curves to the data.

The CRP is an effective and attractive technique for understanding the similarities and dissimilarities represented in the two trajectories in the phase space, and has been applied in many scientific fields such as astrophysics, geophysics, biophysics, and hydrometeorology. Recurrence plot, which was first proposed by Eckmann et al. (1987) and developed later by Zbilut et al. (1998), is a tool for visualizing the recurrent states of nonlinear dynamical systems in the phase space. Its bivariate extension, the so-called CRP, was later applied to determine the phase differences between the two similar systems by comparing their states.

\section{Analysis results}

The left panel of Figure 2 shows the relationship between northern-hemispheric flare index (NHFI) and southernhemispheric flare index (SHFI), and the slope of the straight line fit (the green line shown in this panel) is $0.7726 \pm 0.0483$. Here, 0.7726 is the slope of the fitting line, and 0.0483 is the deviation. The data points of NHFI and SHFI are 588. The right panel of Figure 2 displays the results of cross-correlation analysis of hemispheric flare index, in which the abscissa indicates the leading and lagging shifts of NHFI with respect to SHFI. The green lines shown in the right panel of Figure 2 is the $95 \%$ confidence level. In this case, the phase difference in the two hemispheres is defined as the position of the largest correlation coefficient, and the value is estimated to be 7 months. Therefore, solar $\mathrm{H} \alpha$ flare activity on both hemispheres are highly correlated with each other, but NHFI begins 7 months earlier than SHFI on the considered time interval. Actually, many authors found that solar magnetic structures on both hemispheres are not symmetric and synchronous at any time. For example, by using the wavelet transform method, $\mathrm{Li}$ et al. (2010) found that the difference of solar cycle length could lead to phase asynchrony between the two hemispheres, and flare activity in the northern hemisphere should lead that in the southern one for the low frequency components in the time interval 1966 January-2007 December; using hemispheric sunspot-area and sunspot-number data from solar cycles 12 23, Norton \& Gallagher (2010) found that hemispheric differences range from 0 to 19 months for difference phases in the solar cycles, and the phase shifts in the declining phase are obviously greater than those at the rising phase and maximum. 

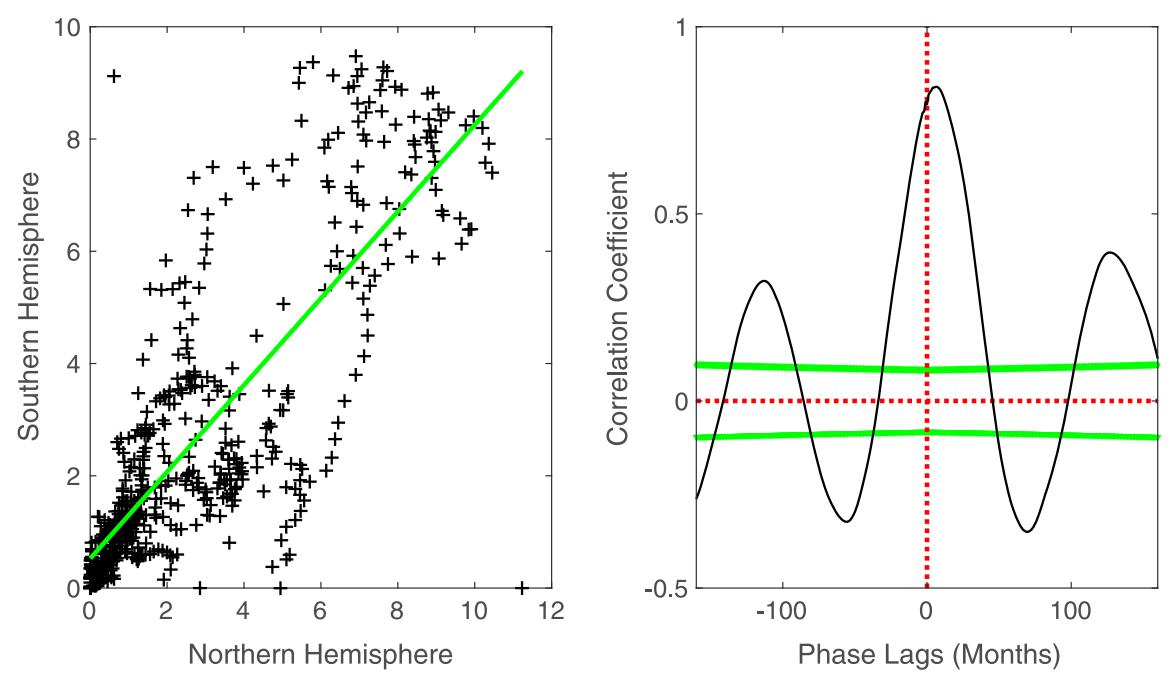

Fig. 2. Left panel: NHFI vs. SHFI at monthly interval from 1966 January to 2014 December. Right panel: cross-correlation analysis between NHFI and SHFI for the whole time interval.
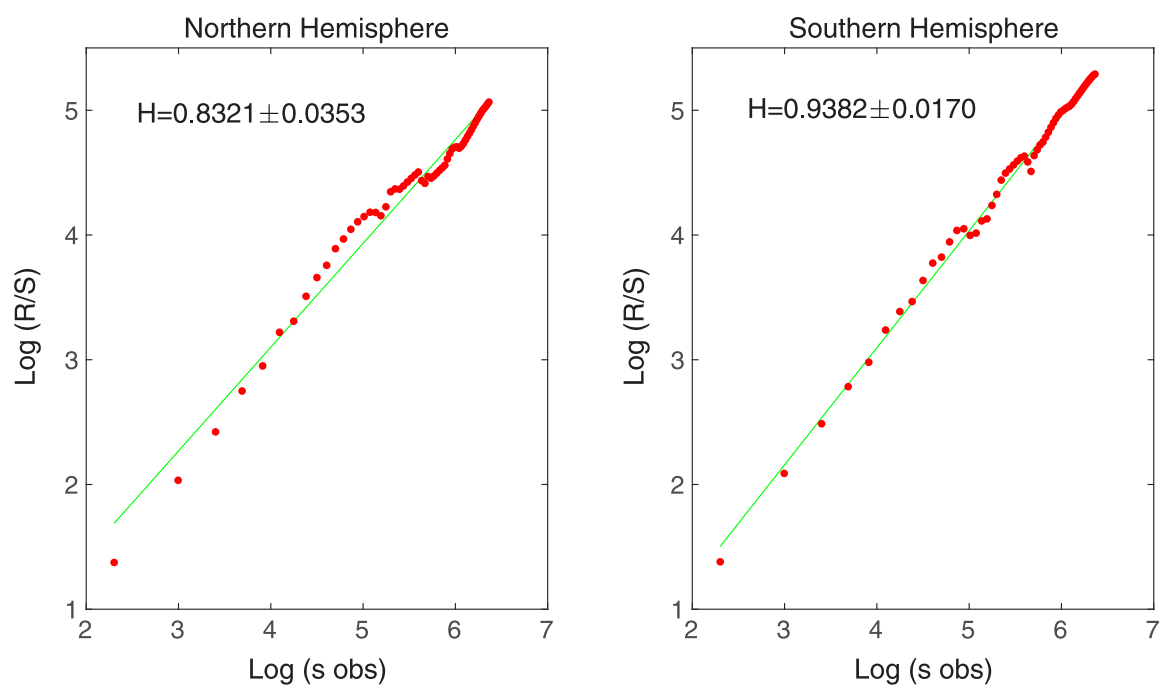

Fig. 3. Log-log plots of the R/S values versus the observational numbers for NHFI (left panel) and SHFI (right panel) respectively. The corresponding linear fitting lines are shown as the green lines.

They also found that cross-hemispheric coupling could be strongest at solar minimum, when there are large quantities of magnetic flux at the Equator. By studying the north-south asymmetry of monthly sunspot areas during the cycle 23 and rising phase of cycle 24, Ravindra \& Javaraiah (2015) found that there is a 5-6 months time delay in the activity levels of two hemispheres.

The $\mathrm{R} / \mathrm{S}$ analysis is non-parametric and is shown to be robust even in the presence of a discrete noise level, in the sense that there are no specific requirements for the distribution of the time series. The R/S values are plotted versus the observational numbers on a log-log graph, and a straight line can be used to fit their linear relationship. The green lines shown in Figure 3 are the linear fitting lines for NHFI (left panel) and SHFI (right panel) respectively. The horizontal and vertical coordinates are the time spans $s$ and the R/S values, respectively. From this figure one can see that the Hurst exponents are estimated to be $H=0.8321 \pm 0.0353$ for NHFI and $H=0.9382 \pm 0.0170$ for SHFI, respectively. It is well known that the Hurst exponent of about 0.5 can be taken as an indication of uncorrelated data set and its value obviously larger than 0.7 indicates the long-range persistence. The analysis results obtained here strongly suggest that long-range persistence indeed exists in solar $\mathrm{H} \alpha$ flare activity on both hemispheres. Besides, the two plots imply that an asymmetric behavior of solar $\mathrm{H} \alpha$ flare activity occurs in the two hemispheres. The Hurst exponents of hemispheric flare activity roughly agree with those reported in the literature by previous authors, whose studies focused on sunspot numbers $(H=0.86 \pm 0.05$, Mandelbrot \& Wallis, 1969; $H=0.87 \pm 0.01$, Kilcik et al., 2009; $H=0.81$, Xapsos \& Burke, 2009; $H=0.8033$, Zhou et al., 2014), sunspot areas $(H=0.7834$, Zhou et al., 2014), solar radius $(H=0.7214 \pm 0.0021$, Kilic \& Golbasi, 2011), H $\alpha$ flare activity 

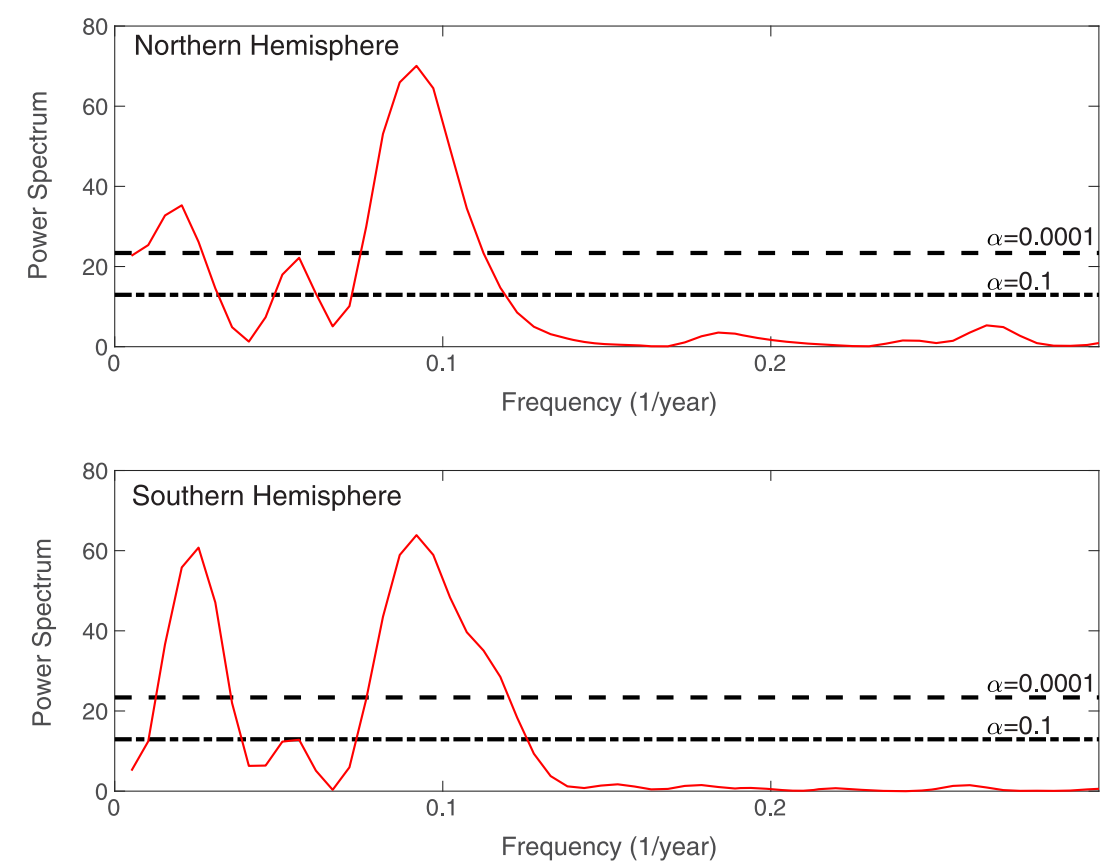

Fig. 4. Lomb-Scargle periodograms of solar $\mathrm{H} \alpha$ flare activity for the northern (upper panel) and southern hemispheres, respectively.

$(H=0.73 \pm 0.01$, Lepreti et al., $2000 ; H=0.7625 \pm 0.0024$, Kilic \& Golbasi, 2011), and cosmogenic radiocarbon data ( $H=0.84$, Ruzmaikin et al., 1994). It should be noted that different authors focused on different solar indices and different time intervals, so the values of the Hurst exponent range from 0.7 to 0.8 with a minor fluctuation.

For calculating the fractal dimension of solar $\mathrm{H} \alpha$ flare activity on both hemispheres, we first apply the linear relationship between the fractal dimension and the Hurst exponent to calculate $D_{1}$, and then the box-counting algorithm is used to compute the fractal dimension $D_{2}$. According to the mathematical relationship $D_{1}=2-H$, the fractal dimensions are calculated to be $D_{1}=1.1679 \pm 0.0353$ for NHFI and $D_{1}=1.0618 \pm 0.0170$ for SHFI, respectively. Based on the boxcounting algorithm presented in Greenside et al. (1982) and Zou et al. (2014), the fractal dimensions $D_{2}$ are found to be $1.473 \pm 0.057$ for NHFI and $1.501 \pm 0.054$ for SHFI, respectively. That is to say, the fractal dimension is not identical, i.e., the $D_{1}$ of NHFI is greater than that of SHFI, whereas the $D_{2}$ of NHFI is smaller than that of SHFI. Actually, the fractal dimension and the Hurst exponent are independent of each other: the former is a local property, and the latter is a global characteristic. Only for the self-affine processes, the local property could be reflected in the global one. In other words, the assumption of statistical self-affinity couples local and global behavior, or fractal dimension and Hurst effect. Therefore, the fractal dimensions derived from the boxcounting algorithm are not statistically compatible with the linear relationship, and solar $\mathrm{H} \alpha$ flare activity is clearly distinct from local roughness properties.

Using the LS periodogram technique, we found a large number of short-term and mid-term periodicities, but many of them are below the $90 \%$ confidence level $(\alpha=0.1)$. Here, different values of $\alpha$ correspond to different confidence levels, for instance, $\alpha=0.1,0.01,0.001$, and 0.0001 correspond to
$90 \%, 99 \%, 99.9 \%$, and $99.99 \%$ confidence levels. It is well known that solar activity indicators display temporal variations from a few days to several years, and the 27 days rotation cycle and the 11 years Schwabe solar cycle are the two remarkable periodicities. In this study, the periodicities smaller than 11 years are defined as the short-term and mid-term periodicities, and only the detected periodicities that are above the $90 \%$ confidence level are taken into account. Figure 4 only displays the low-frequency scales ranging from 0 to 0.3 (1/year), and the high-frequency components larger than 0.3 (1/year) are not taken into account. The periodicities of the first frequency peaks for NHFI and SHFI are 48.92 years and 39.14 years, which are corresponding to the frequency scales of 0.02044 (1/ year) and 0.02555 (1/year) respectively. Because the time span of the two time series analyzed in this work is only 49 years, so these two periodicities can be considered to be a typical feature suggesting the presence of an underlying trend in the time series. The periodicities of the second frequency peaks for both time series are 17.79 years. Earlier studies (Howard \& Labonte, 1980; Leroy \& Noens, 1983) showed that this prominent periodicity could be referred to be the solar full-disk activity cycle, which covers from the maximum of a given solar cycle to the minimum of the following solar cycle. By studying the latitude evolution of coronal data from 1944 to 1974, Leroy \& Noens (1983) arrived at a conclusion that the solar magnetic indices could extend over more than 11 years periodicity, and they provide an increasing evidence that the duration of solar magnetic cycle is greater than the time span between two consecutive solar cycles. There are several lines of evidence that the extended activity cycle is longer than the nominal 11 years Schwabe cycle, and the torsional oscillations reported by Howard \& Labonte (1980) provided evidence for an even longer extended cycle. The latitudinal derivative of the torsional oscillations, corresponding to maximal velocity shear, bisects the wings of the butterfly diagram in each 


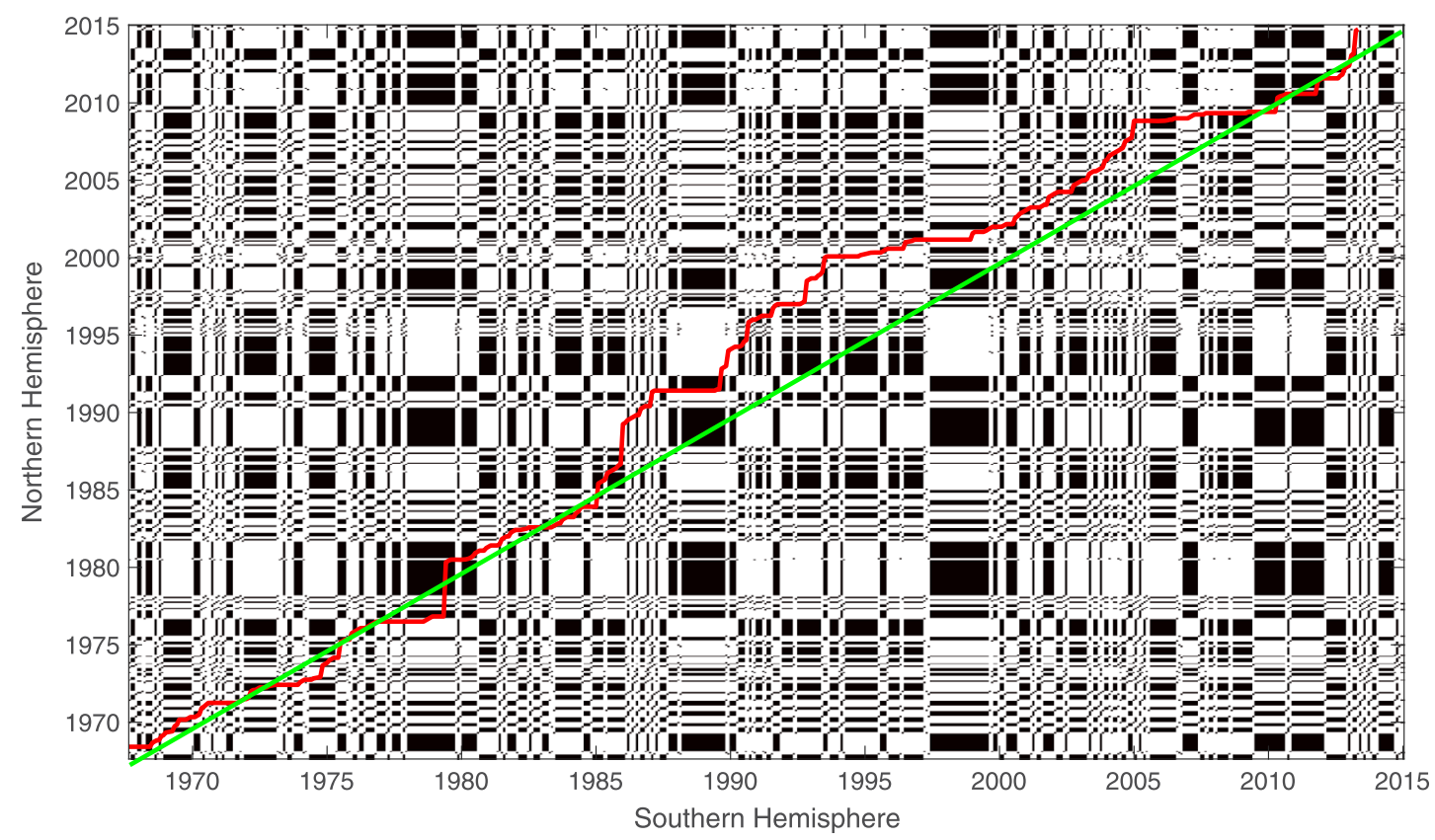

Fig. 5. CRP analysis of solar $\mathrm{H} \alpha$ flare activity on both hemispheres, which is base for estimation of rescaling function. The green and red lines shown in this figure are the main diagonal and the LOS respectively.

hemisphere. Recently, Cliver (2014) reviewed the growth of observational evidence for the extended cycle of solar activity and the Sun's 22 years magnetic cycle, and discussed their role in predicting the amplitude of the following solar cycles. The periodicities of the third frequency peaks for NHFI and SHFI are 10.87 years. There is no doubt that the periodicity of 10.87 years is the well-known 11 years Schwabe cycle. Therefore, the significant periodicities of solar $\mathrm{H} \alpha$ flare activity on both hemispheres are the 17 years full-disk activity cycle and the 11-years Schwabe cycle.

Here, CRP approach is introduced to analyze the dependencies of hemispheric flare index by comparing their phase space states. One of the most important advantage of CRP is that the local difference described by bowed lines of two time series could be well revealed and determined (Stangalini et al., 2017). The CRP pattern of hemispheric solar $\mathrm{H} \alpha$ flare activity is calculated using a varying recurrence threshold $\varepsilon$, preserving a recurrence point density of $10 \%$. For the construction of the LOS (line of synchronization, see Appendix C), the embedding dimension of 3 is used. Earlier studies showed that the dynamical behavior of solar magnetic activity is governed by a low dimensional chaotic attractor, and the embedding dimension is 3 for sunspot numbers and polar faculae (Letellier et al., 2006). The green line shown in Figure 5 is the main diagonal line, which marks the line of identity (LOI). Actually, when the two time series are not absolutely identical, this line becomes somewhat disrupted and is named as LOS. From this point of view, the LOS is not a straight line but is a curve with different amplitudes, which is shown as the red line in the figure. As the LOS could be applied to estimated the non-parametric rescaling behavior between two data sets. From the figure, most points of the LOS are at the left side of the main diagonal line, indicating that NHFI precedes SHFI in phase with several months, especially in the decline phase and minimum of solar cycles. For clearness, we extract the LOS values from the CRP pattern, and the result is shown in Figure 6. The average of all LOS values is calculated to be 8.7 months, implying that the NHFI leads in phase during the total time interval. This result is agreement with (but has a larger value) and further enhances the analysis result given by the cross-correlation analysis. Our results strongly support the point view that the relative phase relation of solar $\mathrm{H} \alpha$ flare activity exhibits obviously hemispheric asynchrony ranging from several months to tens of months. By using CRP technique and extracting LOS, Zolotova et al. (2009) observed a long-term persistence of phase leading in one of the hemispheres, which lasts almost 4 solar cycles and probably corresponds to the Gleissberg cycle. They also found that phase lag in the onsets of sunspot activities on both hemispheres was more than a year and sometimes increased to a maximum of 25 months. However, the hemispheric phase difference derived by Donner \& Thiel (2007), who studied the hemispheric sunspot areas from 1874 to 2007 with the scaleresolved phase coherence analysis, did not exceed 10 months. Actually, using the CRP technique one can compare the time scales of the two data series on a point-by-point basis. However, the wavelet coherence analysis has the disadvantage that it needs to consider sequences of data points, causing an averaging of the property being measured.

\section{Discussion and conclusions}

With the time series of solar $\mathrm{H} \alpha$ flare activity in the northern and southern hemispheres from 1966 January to 2014 December, the statistical properties such as the linear fitting relation, the phase asynchrony, the long-range persistence, and the quasi-periodic variation are studied. The prominent results obtained from several analysis techniques bring out the following conclusions and discussions. 


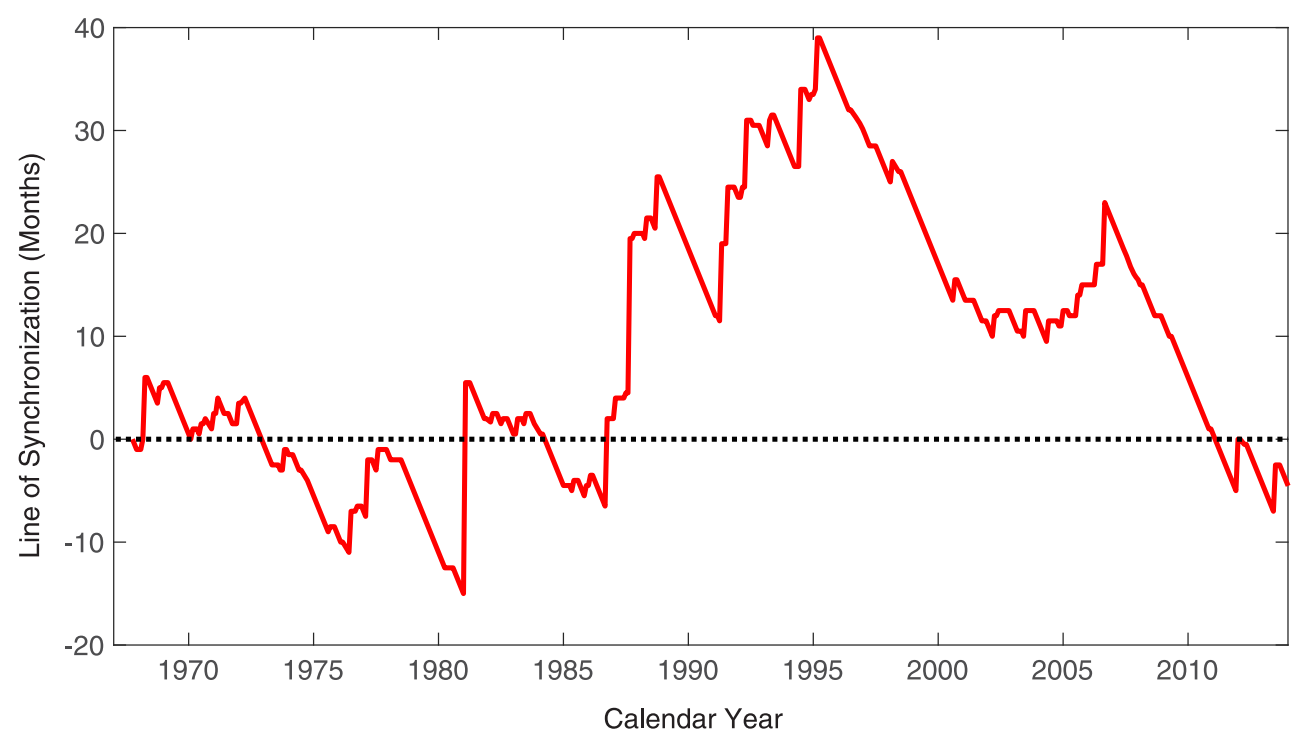

Fig. 6. The LOS values extracted from the CRP pattern to display the more accurate phase relationship.

Cross-correlation analysis simply revealed that solar $\mathrm{H} \alpha$ flare activities in the two hemispheres are highly correlated with each other, but the northern hemisphere begins seven months earlier than the southern one. CRP technique further confirmed this result, but it provides more information on the hemispheric asynchrony. Based on a point-by-point basis, the northern hemisphere is found to precede the southern one with phase shifts ranging from several months to tens of months.

The results derived by the $\mathrm{R} / \mathrm{S}$ analysis strongly support the idea that solar magnetic indicators exhibit long-range persistence, but the dynamical complexity in the two hemispheres are not identical. Recently, Suyal et al. (2009) found that sunspot activity shows a variety of persistent property over shorter and longer time scales corresponding to distinct Hurst exponents, and explained that the multiple values could be caused by the deterministic chaotic attractors which have different centers of rotation. The chaotic motion, observed in deterministic systems such as the Sun, is temporally aperiodic and is strange because it has a fractal geometry. An attractor in a phase space is a region where we have a higher number of points than in another region, i.e., the trajectory passes through this region again and again. The chaotic attractors in the deterministic system is called the deterministic chaotic attractors. Here, it should be noted that the chaotic motion does not refer to the Sun, but to its magnetic activity. Earlier study (Kilcik et al., 2009) showed that the solar activity and space weather prediction in the following solar cycles could be done by combining R/S analysis and Sugihara-May algorithm (Sugihara \& May, 1990). Sugihara-May algorithm is an approach that compares a library of (known) past patterns to patterns seen later in the real time series. It does this by reconstructing an attractor from the library, locating the "present" point on the attractor, and tracing that point forward along the attractor's trajectory. Kilcik et al. (2009) once applied these two techniques to forecast the strength and the maximum of current solar cycle, and found that solar cycle has 87 sunspot units and reaches the maximum in 2012 December.

The quasi-periodic evolutions of various types of solar magnetic indicators have been studied by a large number of authors (Kilcik et al., 2010; Mendoza \& Velasco-Herrera, 2011; Feng et al., 2017), and many of them focus attention on the short-term and mid-term periodicities, such as the Riegertype periodicities and the quasi-biennial oscillations (QBO). However, we only found two typical periodicities that exist in solar $\mathrm{H} \alpha$ activity, maybe the sampled time (one month) of monthly time series is the main reason. In the near future, the daily time series of flare index in different hemispheres and in the whole solar disk should be used to determine their shortand mid-term periodicities by statistical analysis techniques such as empirical mode decomposition or Hilbert-Huang transform. We believe such relatively modern approaches could reveal much more information on the quasi-periodic variations of solar activity indices, which have been proved by Xiang \& Qu (2016), and Feng et al. (2017). But anyway, the most-type periodicities especially the 17 years full-disk cycle of solar $\mathrm{H} \alpha$ activity further suggest that the duration of solar magnetic activity could extend over more than an eleven-years periodicity, which introduced and studied by Leroy \& Noens (1983), and Wilson et al. (1988).

So far, the physical origin of north-south asymmetry of solar magnetic activity is not fully understood, but there are some theoretical explanations from the solar dynamo theories. For example, Jiang et al. (2007) remarked that the hemispheric coupling could be produced by a poloidal large-scale magnetic field formed in the convective zone and trans-equatorial loops in the solar corona as a visible signature of the dynamo process, and the hemispheric coupling could be continuous or intermittent in time, and the last case could result in a strong hemispheric asymmetry. Goel \& Choudhuri (2009) found that the randomness in the Babcock-Leighton dynamo processes could make the poloidal field stronger in one hemisphere than the other one, and this situation usually happens at the end of a solar cycle and this could produce a hemispheric asymmetry in the following cycle. Belucz \& Dikpati (2013) found that the hemispheric asymmetry of solar magnetic activity depends on the differences in the amplitude of the meridional circulations in those hemispheres. Belucz et al. (2015) demonstrated that the effect of greater inflows into active region belts in one 
hemisphere could make the activity level in that hemisphere larger compare to the other hemisphere. They also found that the dominance of one hemisphere could last for more than one solar cycle. Shukuya \& Kusano (2017) investigated the relationship between the dipole- and quadrupole-type components of the magnetic field in the solar cycle using the meanfield theory based on the flux transport dynamo model. They found that there are two different attractors of the solar cycle, in which either the north or the south polar field is first reversed, and that the flux transport dynamo model explains well the phase-asymmetry of sunspot activity and the polar field reversal without any ad hoc source of asymmetry. Blanter et al. (2017) applied a Kuramoto model of coupled oscillators to investigate the north-south asymmetry and properties of meridional circulation, and found that a persistent hemispheric asymmetry of sunspots and the change of the leading hemisphere could indeed both be the result of the evolving frequencies of meridional circulation; the necessary asymmetry of the meridional flow may be small; and the crossequatorial coupling has an intermediate range value. We wish that, in the near future, more observational data driven numerical simulations required to better understand the hemispheric asymmetry and asynchrony of solar magnetic activities.

Acknowledgements. Data sets of solar $\mathrm{H} \alpha$ flare activity in the northern and southern hemispheres applied in this work were calculated by T. Atac and A. Ozguc from Bogazici University Kandilli Observatory, Istanbul, Turkey (http://www.koeri. boun.edu.tr/astronomy/). This work is supported by the Joint Research Fund in Astronomy (U1531140) under cooperative agreement between the National Natural Science Foundation of China (NSFC) and Chinese Academy of Sciences (CAS), the Youth Innovation Promotion Association CAS, the CAS "Light of West China" Program, the Yunnan Science Foundation of China (2015FB192), the open research program of CAS Key Laboratory of Solar Activity (KLSA201709), and the Key Laboratory of Geospace Environment, CAS, University of Science \& Technology of China. Last, but not least, the authors wish to express their gratitude to anonymous referees and the editor for meaningful suggestions and comments that greatly improved the content and presentation of the paper. The editor thanks two anonymous referees for their assistance in evaluating this paper.

\section{References}

Atac T, Ozguc A. 1998. Flare index of solar cycle 22. Sol Phys 180: 397-407. DOI:10.1023/A:1005047805338.

Atac T, Ozguc A. 2006. Overview of the solar activity during solar cycle 23. Sol Phys 233: 139-153. DOI:10.1007/s11207-006-1112-3.

Belucz B, Dikpati M. 2013. Role of asymmetric meridional circulation in producing north-south asymmetry in a solar cycle dynamo model. Astrophys J 779: 4. DOI:10.1088/0004-637X/779/1/4.

Belucz B, Dikpati M, Forgács-Dajka E. 2015. A Babcock-Leighton solar dynamo model with multi-cellular meridional circulation in advection- and diffusion-dominated regimes. Astrophys $J$ 806: 169. DOI:10.1088/0004-637X/806/2/169.

Benz AO. 2008. Flare observations. Living Rev Sol Phys 5: 1. DOI:10.12942/lrsp-2008-1.
Blanter E, Le Mouël J-L, Shnirman M, Courtillot V. 2017. Reconstruction of the north-south solar asymmetry with a Kuramoto model. Sol Phys 292: 54. DOI:10.1007/s11207-0171078-3.

Cliver EW. 2014. The extended cycle of solar activity and the Sun's 22 year magnetic cycle. Space Sci Rev 186: 169-189. DOI:10.1007/ s11214-014-0093-Z.

Deng LH, Xiang YY, Qu ZN, An JM. 2016. Systematic regularity of hemispheric sunspot areas over the past 140 years. Astron $J$ 151: 70. DOI:10.3847/0004-6256/151/3/70.

Donner R, Thiel M. 2007. Scale-resolved phase coherence analysis of hemispheric sunspot activity: a new look at the north-south asymmetry. Astron Astrophys 475: L33-L36. DOI:10.1051/00046361:20078672.

Eckmann JP, Oliffson Kamphorst S, Ruelle D. 1987. Recurrence plots of dynamical systems. EPL (Europhys Lett) 4: 973. DOI:10.1209/ 0295-5075/4/9/004.

Ermolli I, Shibasaki K, Tlatov A, van Driel-Gesztelyi L. 2014. Solar cycle indices from the photosphere to the corona: measurements and underlying physics. Space Sci Rev 186: 105-135. DOI:10.1007/s11214-014-0089-8.

Feng S, Yu L, Yang Y. 2013. The relationship between grouped solar flares and sunspot activity. Bull Astron Soc India 41: 237.

Feng S, Yu L, Wang F, Deng H, Yang Y. 2017. Midterm periodicity analysis of the Mount Wilson magnetic indices using the synchrosqueezing transform. Astrophys $J$ 845: 11. DOI:10.3847/ 1538-4357/aa7d52.

Goel A, Choudhuri AR. 2009. The hemispheric asymmetry of solar activity during the last century and the solar dynamo. Res Astron Astrophys 9: 115-126. DOI:10.1088/1674-4527/9/1/010.

Greenside HS, Wolf A, Swift J, Pignataro T. 1982. Impracticality of a box-counting algorithm for calculating the dimensionality of strange attractors. Phys Rev A 25: 3453-3456. DOI:10.1103/ PhysRevA.25.3453.

Horne JH, Baliunas SL. 1986. A prescription for period analysis of unevenly sampled time series. Astrophys $J$ 302: 757-763. DOI:10.1086/164037.

Howard R, Labonte BJ. 1980. The sun is observed to be a torsional oscillator with a period of 11 years. Astrophys J Lett 239: L33-L36. DOI:10.1086/183286.

Hurst HE. 1951. Long-term storage capacity of reservoirs. Trans Am Soc Civil Eng 116: 770-808.

Jiang J, Choudhuri AR, Wang J. 2007. A new explanation for the origin of trans-equatorial loops based on a dynamo model. Sol Phys 245: 19-25. DOI:10.1007/s11207-007-9033-3.

Joshi B, Joshi A. 2004. The north-south asymmetry of soft X-ray flare index during solar cycles 21, 22 and 23. Sol Phys 219: 343-356. DOI:10.1023/B:SOLA.0000022977.95023.a7.

Joshi B, Bhattacharyya R, Pandey KK, Kushwaha U, Moon YJ. 2015. Evolutionary aspects and north-south asymmetry of soft X-ray flare index during solar cycles 21, 22, and 23. Astron Astrophys 582: A4. DOI:10.1051/0004-6361/201526369.

Kilcik A, Anderson CNK, Rozelot JP, Ye H, Sugihara G, Ozguc A. 2009. Nonlinear prediction of solar cycle 24. Astrophys $J$ 693: 1173-1177. DOI:10.1088/0004-637X/693/2/1173.

Kilcik A, Özgüç A, Rozelot JP, Ataç T. 2010. Periodicities in solar flare index for cycles 21-23 revisited. Sol Phys 264: 255-268. DOI:10.1007/s11207-010-9567-7.

Kilic H, Golbasi O. 2011. Comparison of long-term trend of solar radius with sunspot activity and flare index. Astrophys Space Sci 334: 75-81. DOI:10.1007/s10509-011-0714-x.

Kleczek J. 1952. Ionospheric disturbances and flares in the 11 years cycle. Bull Astron Inst Czechoslov 3: 52. 
Lepreti F, Fanello PC, Zaccaro F, Carbone V. 2000. Persistence of solar activity on small scales: Hurst analysis of time series coming from $\mathrm{H} \alpha$ flares. Sol Phys 197: 149-156. DOI:10.1023/ A: 1026560212716.

Leroy JL, Noens JC. 1983. Does the solar activity cycle extend over more than an 11 year period? Astron Astrophys 120: L1.

Letellier C, Aguirre LA, Maquet J, Gilmore R. 2006. Evidence for low dimensional chaos in sunspot cycles. Astron Astrophys 449: 379-387. DOI:10.1051/0004-6361:20053947.

Li M. 2010. Fractal time series - a tutorial review. Math Probl Eng 2010: 157264.

Li KJ, Gao PX, Zhan LS, Shi XJ, Zhu WW. 2010. Relative phase analyses of long-term hemispheric solar flare activity. Mon Not $R$ Astron Soc 401: 342-346. DOI:10.1111/j.1365-2966.2009.15639.x.

Lomb NR. 1976. Least-squares frequency analysis of unequally spaced data. Astrophys Space Sci 39: 447-462. DOI:10.1007/BF00648343.

Mandelbrot BB, Wallis JR. 1969. Some long-run properties of geophysical records. Water Resour Res 5: 321-340. DOI:10.1029/ WR005i002p00321.

Marwan N, Carmen Romano M, Thiel M, Kurths J. 2007. Recurrence plots for the analysis of complex systems. Phys Rep 438: 237-329. DOI:10.1016/j.physrep.2006.11.001.

Mendoza B, Velasco-Herrera VM. 2011. On mid-term periodicities in sunspot groups and flare index. Sol Phys 271: 169-182. DOI:10.1007/s11207-011-9802-x.

Norton AA, Gallagher JC. 2010. Solar-cycle characteristics examined in separate hemispheres: phase, gnevyshev gap, and length of minimum. Sol Phys 261: 193-207. DOI:10.1007/s11207-009-9479-6.

Norton AA, Charbonneau P, Passos D. 2014. Hemispheric coupling: comparing dynamo simulations and observations. Space Sci Rev 186: 251-283. DOI:10.1007/s11214-014-0100-4.

Ouyang Y, Zhou YH, Chen PF, Fang C. 2017. Chirality and magnetic configurations of solar filaments. Astrophys $J$ 835: 94. DOI:10.3847/1538-4357/835/1/94.

Ozguc A, Atac T, Rybak J. 2003. Temporal variability of the flare index (1966-2001). Sol Phys 214: 375-396. DOI:10.1023/ A: 1024225802080.

Ravindra B, Javaraiah J. 2015. Hemispheric asymmetry of sunspot area in solar cycle 23 and rising phase of solar cycle 24: comparison of three data sets. New Astron 39: 55-63. DOI:10.1016/j. newast.2015.03.004.

Ruzmaikin A, Feynman J, Robinson P. 1994. Long-term persistence of solar activity. Sol Phys 149: 395-403. DOI:10.1007/BF00690625.

Scargle JD. 1982. Studies in astronomical time series analysis. II Statistical aspects of spectral analysis of unevenly spaced data. Astrophys $J$ 263: 835-853. DOI:10.1086/160554.

Shen Y, Liu Y. 2012. Evidence for the wave nature of an extreme ultraviolet wave observed by the atmospheric imaging assembly on board the solar dynamics observatory. Astrophys $J$ 754: 7. DOI:10.1088/0004-637X/754/1/7.

Shen YD, Liu Y, Liu R. 2011. A time series of filament eruptions observed by three eyes from space: from failed to successful eruptions. Res Astron Astrophy 11: 594-606. DOI:10.1088/16744527/11/5/009.

Shetye J, Tripathi D, Dikpati M. 2015. Observations and modeling of north-south asymmetries using a flux transport dynamo. Astrophys $J$ 799: 220. DOI:10.1088/0004-637X/799/2/220.
Shibata K, Magara T. 2011. Solar flares: magnetohydrodynamic processes. Living Rev Sol Phys 8: 6. DOI:10.12942/lrsp-2011-6.

Shukuya D, Kusano K. 2017. Simulation study of hemispheric phaseasymmetry in the solar cycle. Astrophys $J$ 835: 84 . DOI:10.3847/ $1538-4357 / 835 / 1 / 84$.

Stangalini M, Ermolli I, Consolini G, Giorgi F. 2017. Recurrence quantification analysis of two solar cycle indices. $J$ Space Weather Space Clim 7: A5. DOI:10.1051/swsc/2017004.

Sugihara G, May RM. 1990. Nonlinear forecasting as a way of distinguishing chaos from measurement error in time series. Nature 344: 734-741. DOI:10.1038/344734a0.

Suyal V, Prasad A, Singh HP. 2009. Nonlinear time series analysis of sunspot data. Sol Phys 260: 441-449. DOI:10.1007/s11207-0099467-x.

Svalgaard L, Kamide Y. 2013. Asymmetric solar polar field reversals. Astrophys $J$ 763: 23. DOI:10.1088/0004-637X/763/1/23.

Temmer M, Veronig A, Hanslmeier A, Otruba W, Messerotti M. 2001. Statistical analysis of solar H $\alpha$ flares. Astron Astrophys 375: 10491061. DOI:10.1051/0004-6361:20010908.

Wilson PR, Altrocki RC, Harvey KL, Martin SF, Snodgrass HB. 1988. The extended solar activity cycle. Nature 333: $748-750$. DOI:10.1038/333748a0.

Xapsos MA, Burke EA. 2009. Evidence of 6000 year periodicity in reconstructed sunspot numbers. Sol Phys 257: 363-369. DOI:10.1007/s11207-009-9380-3.

Xiang NB, Qu ZN. 2016. Ensemble empirical mode decomposition of the magnetic field of the Sun as a star. Astron $J$ 151: 76. DOI:10.3847/0004-6256/151/3/76.

Xie JL, Shi XJ, Xu JC. 2012. Temporal variation of hemispheric solar rotation. Res Astron Astrophys 12: 187-200. DOI:10.1088/16744527/12/2/007

Yan XL, Qu ZQ, Xu CL. 2008. A statistical study on rotating sunspots: polarities, rotation directions, and helicities. Astrophys $J$ Lett 682: L65. DOI:10.1086/590953.

Yan XL, Xue ZK, Pan GM, Wang JC, Xiang YY, Kong DF, Yang LH. 2015. The formation and magnetic structures of active-region filaments observed by NVST, SDO, and hinode. Astrophys J Suppl Ser 219: 17. DOI:10.1088/0067-0049/219/2/17.

Zbilut JP, Giuliani A, Webber CL Jr. 1998. Detecting deterministic signals in exceptionally noisy environments using cross-recurrence quantification. Phys Lett A 246: 122-128. DOI:10.1016/S03759601(98)00457-5.

Zhang L, Mursula K, Usoskin I. 2015. Solar surface rotation: N-S asymmetry and recent speed-up. Astron Astrophys 575: L2. DOI:10.1051/0004-6361/201425169.

Zhou S, Feng Y, Wu WY, Li Y, Liu J. 2014. Low-dimensional chaos and fractal properties of long-term sunspot activity. Res Astron Astrophy 14: 104-112. DOI:10.1088/1674-4527/14/1/009.

Zolotova NV, Ponyavin DI. 2007. Synchronization in sunspot indices in the two hemispheres. Sol Phys 243: 193-203. DOI:10.1007/ s11207-007-0405-5.

Zolotova NV, Ponyavin DI, Marwan N, Kurths J. 2009. Long-term asymmetry in the wings of the butterfly diagram. Astron Astrophys 503: 197-201. DOI:10.1051/0004-6361/200811430.

Zou P, Li QX, Wu N. 2014. Non-linear analysis of the long-term behaviour of solar filaments. Mon Not R Astron Soc 437: 38-45. DOI:10.1093/mnras/stt1868. 


\section{Appendices}

\section{Appendix A: Rescaled range analysis}

The $\mathrm{R} / \mathrm{S}$ analysis approach is performed on the discrete time series $x(i)$ of dimension $n$ by calculating three factors: first the average value $\bar{x}$ of $x(i)$, second the $R(s)$, the series of the range of cumulative deviates over the time span $s$, and third the $S(s)$, the series of the standard deviations over the time span $s$. The $R(s)$ and $S(s)$ could be given by the following formulas:

$$
\begin{gathered}
R(s)=\operatorname{Max}\left\{\sum_{i=1}^{s} x(i)-\bar{x}\right\}-\operatorname{Min}\left\{\sum_{i=1}^{s} x(i)-\bar{x}\right\}, \\
S(s)=\left\{\frac{1}{s} \sum_{i=1}^{s}[x(i)-\bar{x}]^{2}\right\}^{\frac{1}{2}}, \quad s=1, \ldots, n
\end{gathered}
$$

The ratio between $R$ and $S$ could be described the long-range persistence of a time series by the experimental relationship $\langle R / S\rangle=s^{H}$, where $H$ is the Hurst exponent. In the log-log graph of $R / S$ values versus the time spans $s$, the value of $H$ is equal to the slope of the fitting line. For $H$ equals to 0.5 the time series represents a random walk or uncorrelated. The value of $0<H<0.5$ indicates that it exhibits an anti-persistence behavior, i.e., its trend will likely reverse in the future. The value of $H$ lying between 0.5 and 1 implies persistent time series characterized by long-range effects, i.e., the successive increments are positively correlated with the preceding observations (Suyal et al., 2009).

For a time series, one can use the Hurst exponent and the fractal dimension to describe its global property and the local one, respectively. From a statistical point of view, the fractal dimension is a statistical quantity that measures the dynamical complexity of a given time series. In general, a fractal time series may not be globally self-similar. Nevertheless, a series that is not self-similar maybe locally self-similar. If the given signal is a self-similar process, the Hurst exponent $H$ of a time series is directly related to its fractal dimension $D_{1}$, and the mathematical relationship between these two parameters is $D_{1}=2-H$. Various analysis techniques regarding the estimation of the fractal dimension could be summarized as autocorrelation function regression, periodogram regression method, generalized linear regression, scaled and rescaled windowed variance methods, maximum likelihood estimation method, fractional Fourier transform, and so on. The review article of $\mathrm{Li}$ (2010) gives a tutorial review about these approaches. Here, we apply the box-counting algorithm to compute the fractal dimension $D_{2}$. To apply the box-counting scheme, one should first construct a delay embedding attractor from the time series in an embedding space of m-dimension with a suitably chosen time delay, and then try to cover the attractor using m-dimensional cubes of side length $r$. The fractal dimension $D_{2}$ is then defined by the expression:

$$
D_{2}=\lim _{r \rightarrow 0} \frac{\log C(r)}{\log (r)},
$$

with $C(r)$ given by

$$
C(r)=\sum_{i=1}^{N(r)} p_{i}^{2}(r)
$$

Here $N(r)$ is the number of non empty boxes and $p_{i}(r)$ is the probability that the trajectory passes through the $i$ th box. But for a finite data stream, the limiting value is not available and hence a proper linear part in the $\log C(r)$ versus $\log r$ plot is identified as the scaling region whose slope is taken to be $D_{2}$. Conventionally one takes $p_{i}(r)=m_{i} / N_{p}$ as the weight of the $i$ th box, where $N_{p}$ is the total number of points in the attractor and $m_{i}$ is the number of points falling in the $i$ th box.

\section{Appendix B: Lomb-Scargle periodogram}

The mean $\bar{x}$ and the variance $\sigma^{2}$ of $x_{i}$ can be defined as $\bar{x}=1 / n \sum_{i=1}^{n} x_{i}$, and $\sigma^{2}=1 /(n-1) \sum_{i=1}^{n}\left(x_{i}-\bar{x}\right)^{2}$, then the Lomb-Scargle periodogram of frequency $(\omega=2 \pi f)$ is expressed as:

$$
\begin{gathered}
P_{n}(\omega)=\left(\frac{1}{2 \sigma^{2}}\right) \times\left\{\frac{\left[\sum_{i=1}^{n}\left(x_{i}-\bar{x}\right) \cos \omega\left(t_{i}-\tau\right)\right]^{2}}{\sum_{i=1}^{n} \cos ^{2} \omega\left(t_{i}-\tau\right)}\right. \\
\left.+\frac{\left[\sum_{i=1}^{n}\left(x_{i}-\bar{x}\right) \sin \omega\left(t_{i}-\tau\right)\right]^{2}}{\sum_{i=1}^{n} \sin ^{2} \omega\left(t_{i}-\tau\right)}\right\}
\end{gathered}
$$

Here $\tau$ is calculated from the relation:

$$
\tan (2 \omega \tau)=\frac{\sum_{i} \sin 2 \omega t_{i}}{\sum_{i} \cos 2 \omega t_{i}}
$$

Being quite powerful for checking and estimating the significance of weak cyclical components through false alarm probability (FAP), it is easy to obtain the significance of the peak height in the power spectrum. According to Scargle (1982) and Horne \& Baliunas (1986), the FAP that estimates the statistical significance of a peak in the power spectrum is given by $F=1-[1-\exp (-\mathrm{z})]^{n}$, where $z$ is the height of the peak in the normalized power spectrum and $N$ is the number of independent frequencies. The value of the FAP statistical significance can be considered as the different significance levels $(\alpha)$.

The exponential probability distribution function of power, $P_{n}(\omega)$, helps us through FAP to estimate the probability that a given peak is a true signal or whether it is the result of randomly distributed noise. For this periodogram, the probability that the value $P_{n}(\omega)$ is greater than $z$ by chance is given by $\operatorname{Pr}\left[P_{n}(\omega) \geq z\right]=\exp (-z)$.

\section{Appendix C: Cross-recurrence plot}

The CRP could be considered as a generalization of the linear cross-correlation function, and analyzes the parallel occurrence of recurrent states as described by the time series $x_{i}$ and $y_{i}$. The mathematical definition of the cross-recurrence matrix $C R$ could de described as: 


$$
C R_{i, j}^{m, \varepsilon_{i}}=\Theta\left(\varepsilon_{i}-\left\|x_{i}-y_{j}\right\|\right)
$$

where $x_{i}, y_{j} \in R^{m}\left(i=1, \ldots, n_{x}, j=1, \ldots, n_{y}\right), \varepsilon_{i}$ is the threshold distance, $\|\cdot\|$ is the norm (e.g., the Euclidian norm), and $\theta$ is the Heaviside function. Visualization of CRP is a graphi graphical pattern of the matrix $n_{x} \times n_{y}$ and all elements $\left(C R_{i, j}\right)$ of which are either zero or one. Note that both systems are represented in the same phase space, because a CRP looks for those times when a state of the first system recurs to one of the other system. Using experimental data, it is often necessary to reconstruct the trajectories in phase space. If the embedding parameters are estimated from both time series, but are not equal, the higher embedding should be chosen. However, the data under consideration should be from the same (or a very comparable) process and, actually, should represent the same observable. Therefore, the reconstructed phase space should be the same.

An important advantage of the CRP pattern is that it can reveal the local difference of the dynamical evolution of close trajectory segments, represented by bowed lines. A time dilatation or time compression of one of the trajectories causes a distortion of the diagonal lines. Assuming two identical trajectories, the CRP pattern contains the main black diagonal or LOI. If the values of the second trajectory are slightly modified, the LOI will become somewhat disrupted and is called line of synchronisation (LOS). However, if we do not modify the amplitudes but stretch or compress the second trajectory slightly, the LOS will still be continuous but not a straight line with slope one (angle of $\pi / 4$ ). This line can rather become bowed, and the local slope of lines in the CRP pattern corresponds to the transformation of the time axes of the two considered trajectories. A phase difference between the trajectories causes a dislocation of the LOS. Hence, the LOS may lie rather far from the main diagonal of the CRP. Therefore, in the common non-stationary case, the off-set of the LOS away from the main diagonal is an indication of the phase differences between two time series.

The construction of the LOS with the CRP can be performed in different ways. An important requirement is that for each point on the $x$-axis of the CRP a corresponding point on the $y$-axis should be found. Another criteria to obtain a suitable LOS is that the amount of targeted recurrence points by the LOS should converge to the maximum and the amount of gaps in the LOS should converge to the minimum. For this purpose, we will apply a simple two-step algorithm proposed in Marwan et al. (2007), available in the CRP toolbox (http://tocsy.pik-potsdam.de/ CRPtoolbox/) for Matlab software. This algorithm provided a trade-off between efficiency and correctness. The detailed algorithms and steps for constructing the CRP pattern and for calculating the LOS value can be found in the paper of Zolotova et al. (2009).

Cite this article as: Deng L, Zhang X, An J, Cai Y. 2017. Statistical properties of solar H $\alpha$ flare activity. J. Space Weather Space Clim. 7: A34 